\title{
La actividad física durante la internación psiquiátrica en la Ciudad Autónoma de Buenos Aires
}

\author{
Physical Activity in Psychiatric inpatient Institutions In Ciudad de Buenos Aires
}

\section{María Ivana Smoljanovich'}

\begin{abstract}
Resumen
Introducción: La evidencia sobre los beneficios de la actividad física como herramienta en la prevención y tratamiento de patologías psiquiátricas es creciente. El objetivo de esta investigación es conocer la implementación de la actividad física como recurso terapéutico durante las internaciones psiquiátricas de adultos en la Ciudad Autónoma de Buenos Aires. Metodología: Se diseñó una encuesta para obtener información de cada institución con internación psiquiátrica de adultos de esta ciudad con el fin de conocer: si se brindaba o no actividad física a los pacientes durante el período de internación, los motivos, la frecuencia, características y duración de la actividad así como los beneficios observados y/o conocidos sobre el tema. Resultados: El 7l\% de las instituciones brinda actividad física a sus pacientes, nueve son privadas y tres son públicas. La adherencia es mayor en las instituciones privadas. La falta de recursos y el hecho de no considerarla de importancia terapéutica se encuentran entre los motivos por los cuales no se utiliza como herramienta terapéutica. Conclusiones: La actividad física debiera ser incluida dentro de las propuestas terapéuticas en la internación psiquiátrica, la mayoría de las instituciones así lo realizan.
\end{abstract}

Palabras clave: Actividad física - Internación psiquiátrica - Psiquiatría - Ejercicio físico - Pacientes psiquiátricos.

\section{Abstract}

There is growing evidence on the benefits of physical activity as a tool in treatment and prevention of psychiatric pathologies. The goal of this research is to obtain information on the implementation of physical activity as a therapeutic resource during the psychiatric hospitalization of adults in Ciudad de Buenos Aires. Methodology: A survey was designed to obtain information from each Inpatient adult institution in the aforementioned City on: whether or not physical activity was provided to patients during hospitalization, the reasons, the frequency, characteristics and duration of the activity as well as the benefits observed and / or known by the professionals who answered the survey. Results: $7 / \%$ of the institutions provide physical activity to their patients, nine are private and three are public. Adherence is higher in private institutions. The lack of resources and the fact that it is not considered important in psychiatric treatment are among the reasons why they consider that physical activity is not used as a therapeutic tool. Conclusions: Physical activity should be included in the therapeutic tools during psychiatric hospitalization, most of the institutions do include it.

Keywords: Physical activity - Psychiatric inpatient - Physical exercise - Psychiatric hospitalization.

RECIBIDO 15/2/202I - ACEPTADO 7/6/2021

1.Médica diplomada en Neurodesarrollo.

Correspondencia:

ivana_smoljanovich@hotmail.com 


\section{Introducción}

Existe una creciente evidencia sobre la relación entre actividad física y salud mental (Svatkova et al., 2015) como también son cada vez más amplios y prometedores los actuales conocimientos sobre la relación beneficiosa entre actividad física y diversas patologías mentales (Kandola et al., 2016; Svatkova et al., 2015).

Estudios en pacientes con enfermedad de Alzheimer temprana muestran mejoría de la aptitud cardiorrespiratoria relacionada con el ejercicio, asociado con un mejor desempeño de la memoria y una menor atrofia del hipocampo (Morris et al., 2017).

La actividad física regular puede disminuir el riesgo de un episodio depresivo como así también mejorar los síntomas depresivos (Adams et al., 2015; Netz, 2017). Existe evidencia de que la actividad física sumada al tratamiento antidepresivo convencional incrementa la eficacia de dichos tratamientos (Kandola et al., 2016).

Ante situaciones traumáticas que puedan constituir un trastorno, particularmente en el trastorno de estrés post traumático (TEPT), la realización de actividad física disminuye la sintomatología. Dentro de los trastornos por angustia, en pacientes con agorafobia y trastorno de pánico el ejercicio físico aeróbico junto a TCC disminuye los síntomas. Por otro lado, la inactividad física es un factor de riesgo para el desarrollo de ansiedad (Kandola et al., 2018).

Realizar actividad física aporta beneficios en relación con los síntomas negativos de la esquizofrenia, los que suelen ser persistentes y difíciles de tratar. El pobre entrenamiento aeróbico representa un factor de riesgo modificable para la disfunción neurocognitiva en personas con esquizofrenia (Kimhy et al., 2015). Resultados beneficiosos también se observan en pacientes con un primer episodio psicótico que realizan actividad física (Firth et al., 2018).

El estilo de vida sedentario de las personas con esquizofrenia que viven en una institución es a largo plazo una carga para la salud pública. Una intervención simple en el estilo de vida puede ayudar a preparar a las personas para reintegrarse en la sociedad (Loh et al., 2015).

Existen además efectos beneficiosos no psiquiátricos de la realización de actividad física aeróbica en pacientes que padecen patologías psiquiátricas internados, entre los se encuentran la disminución del tiempo de tránsito colónico (el tránsito colónico lento estaría relacionado con una disminución de nutrientes y de absorción de medicación), mejoría de los valores de presión sanguínea arterial y mejoría en la resistencia cardiorrespiratoria (Kim et al., 2014; Song et al., 2018).
En el año 2016 se publicó un artículo sobre la implementación de los programas de actividad física en los hospitales psiquiátricos suizos de habla alemana (Brand et al., 2016). En él se muestra una encuesta donde el $100 \%$ de los encuestados respondieron que los programas de ejercicios y actividad física eran una modalidad de tratamiento integrado del hospital durante la internación.

Todos los participantes refirieron que en su opinión observaron mejorías en el estado físico y psicológico de los pacientes.

La actitud del personal de salud con respecto a la actividad física es un tema importantísimo en este contexto. En otro trabajo publicado en el año 2018 estudiaron la actividad física de pacientes de tres clínicas de Suiza de habla alemana encontrando que $57 \%$ de los pacientes que participaron cumplió con los niveles recomendados de actividad física aeróbica ( $\geq 150 \mathrm{~min}$ de actividad física moderada a vigorosa por semana), superando lo esperado por los autores y siendo un porcentaje similar al de la población Suiza en general. Si bien los motivos no son resultados concluyentes del estudio, los autores sugieren que el motivo por el que dicha tasa fue mayor a la esperada y a la encontrada en otro estudio (Brand et al., 2016) podría ser que las clínicas de donde se obtuvieron los participantes para este trabajo son conscientes de los beneficios potenciales de la actividad física y el ejercicio, y dos de estas clínicas habían participado previamente de otro estudio en el que se comprobó la eficacia del ejercicio y la terapia deportiva (Ehrbar et al., 2018).

Tomando al hombre como una unidad biopsicosocial, en función de la creciente evidencia a favor de los beneficios de la actividad física como herramienta en la prevención y tratamiento de patologías psiquiátricas, y dado que no existe en la bibliografía información sobre la implementación de la actividad física en el período de internación psiquiátrica en la Ciudad Autónoma de Buenos Aires, se diseñó el presente trabajo con el objetivo de conocer la proporción sobre el total de instituciones con internación psiquiátrica de la Ciudad Autónoma de Buenos Aires que brindan algún tipo de actividad física a sus pacientes durante el período de internación, a fin de inferir en qué medida los conocimientos actuales están siendo implementados, los resultados de la experiencia en los casos donde estén siendo utilizados y de no ser así, los motivos de su falta de implementación; a la vez que proporcionar información para la realización de futuros estudios sobre el tema. 


\section{Materiales y métodos}

En mayo de 2018 se realizó un listado de las instituciones públicas y privadas con servicio de internación psiquiátrica de adultos de la Ciudad Autónoma de Buenos Aires. El listado de instituciones públicas (ver anexo 1) se obtuvo de la Guía de Recursos y Derivación en Salud Mental 2014/2015 de la Dirección General de Salud Mental del Ministerio de Salud del Gobierno de la Ciudad de Buenos Aires y la colaboración de profesionales de del capítulo de Psiquiatras en Formación perteneciente a la Asociación de Psiquiatras Argentinos. El listado de instituciones privadas (ver anexo 2) fue reconstruido en base a las instituciones privadas con internación psiquiátrica para adultos que figuran en Internet y la colaboración de profesionales de del capítulo de Psiquiatras en Formación perteneciente a la Asociación de Psiquiatras Argentinos.

Se diseñó una encuesta en formato digital. Se contactó un representante de cada institución de los anexos 1 y 2 . Cada representante trabajaba en la institución a la que representaba. Los representantes contactados fueron en su totalidad médicos, conocidos por la autora o bien fueron sugeridos por profesionales conocidos por la autora. Cada representante fue contactado por la autora informándosele sobre los objetivos del trabajo y consultados sobre su intención de participar. Luego de obtener una respuesta afirmativa sobre su intención de participar se les solicitó una dirección de correo electrónico. A cada participante se le envió un e-mail con una breve descripción de los objetivos del estudio y se adjuntó un link para acceder a la encuesta en formato digital de Google Drive.

- Se les solicitó confirmación una vez completada la encuesta.

- Las encuestas fueron enviadas y respondidas entre los meses de mayo 2018 y mayo 2019.

- Fueron contactados profesionales representantes de todas las instituciones.

- Los resultados fueron almacenados y computados en la aplicación Google Drive.

- La realización de la encuesta fue voluntaria y anónima.

\section{Consideraciones éticas}

La encuesta implicó un riesgo mínimo para los pacientes. No fue realizada a pacientes, ni incluye datos personales de los mismos. La realización de la encuesta fue voluntaria y anónima.

\section{Análisis estadístico}

Se aplicó estadística descriptiva.

\section{Resultados}

Se recibieron un total de diecisiete respuestas (94\%) sobre un total de dieciocho instituciones públicas y privadas. Siete respuestas fueron provenientes de instituciones públicas (sobre un total de siete instituciones públicas según anexo 1) y diez respuestas de instituciones privadas (sobre un total de once instituciones privadas según anexo 2).

\section{ANEXO I}

Guía de instituciones públicas con internación psiquiátrica para adultos en la Ciudad Autónoma de Buenos Aires:

I. Hospital General de Agudos "Dr. Teodoro Alvarez".

2. Hospital de Emergencias Psiquiátricas "Torcuato de Alvear".

3. Hospital Interdisciplinario Psicoasistencial "Dr. José T. Borda”.

4. Hospital Neuropsiquiátrico "Dr. Braulio A. Moyano".

5. Hospital General de Agudos "Dr. Parmenio Piñero".

6. Hospital de Clínicas "José de San Martín”.

7. Hospital General de Agudos “Dr. Ignacio Pirovano”.

\section{ANEXO 2}

Guía de instituciones privadas con internación psiquiátrica para adultos en la Ciudad Autónoma de Buenos Aires:

I. Clínica "Avril”.

2. Clínica "Dharma".

3. Clínica Flores.

4. Clínica "Las Heras".

5. Clínica "Moravia".

6. Clínica "Santa Rosa".

7. Comunidad Terapéutica "Gradiva".

8. Hospital Italiano de Buenos Aires.

9. Instituto Frenopático.

10. Instituto Neurociencias Buenos Aires (INEBA).

I I. Clínica "Témpora".

Todos los participantes de la encuesta fueron médicos. Participaron médicos y psiquiatras de guardia y de planta, residentes de psiquiatría, un jefe de residentes y un jefe de sala de internación.

El rango de cantidad de camas de internación de cada institución es amplio, desde 8 a 500 camas. El 76\% de las instituciones (13 de 17) cuenta con una cantidad de camas entre 17 y 60, un 6\% ( 1 de 17) cuenta con 8 camas y un 18\% (3 de 17) cuenta con más de cien camas.

Catorce de diecisiete encuestados respondieron que en su institución sí se brinda algún tipo de actividad física a los pacientes durante el período de internación psiquiátrica. En dos encuestas con respuestas afirmativas, la descripción de la actividad correspondía a actividades en las cuales el movimiento físico no 
era el objetivo principal (como por ejemplo lectura, musicoterapia y terapia ocupacional) por lo cual esas respuestas no fueron incluidas como afirmativas y fueron incluidos en el grupo que no brinda actividad física a pacientes psiquiátricos internados.

Por lo tanto, el total fue de doce respuestas afirmativas (71\%), mientras que la suma total de respuestas negativas fue de cinco (29\%).

De las doce instituciones que brinda algún tipo de actividad física a sus pacientes, nueve son privadas (75\%) y tres son públicas (25\%) mientras que de las cinco instituciones que no la brindan, cuatro son públicas $(80 \%)$ y una privada (20\%).

A los encuestados que respondieron que sí se brinda actividad física, se les preguntó sobre los motivos por los cuales se realiza la implementación de actividad física. Se trató de una pregunta con opción múltiple y cada encuestado podía optar por más de una opción. Sobre un total de doce respuestas afirmativas, Recreación fue seleccionado por once encuestados (92\%), Por los beneficios en el tratamiento psiquiátrico fue seleccionado por nueve encuestados (75\%) y Por los beneficios cardiovasculares por ocho encuestados (67\%). Dos encuestados respondieron solamente por Recreación, de los cuales uno representaba institución pública y otro a institución privada; un encuestado representante de institución privada respondió únicamente Por sus beneficios en el tratamiento psiquiátrico. Dos encuestados seleccionaron también la opción Otros y describieron por motivos de socialización.

De las doce instituciones que brindan actividad física, cuatro brindan sólo una actividad $(33,3 \%)$ de las cuales tres son privadas y una pública, cuatro brindan dos actividades $(33,3 \%)$, de las cuales tres son privadas y una pública y cuatro brindan más de dos actividades $(33,3 \%)$ de las cuales tres son privadas y una pública.

La frecuencia semanal varía desde una vez por semana ( $8 \%$ correspondiendo a una institución pública) a siete veces ( $8 \%$ correspondiendo a una institución privada). Dentro de este rango hay seis instituciones que brindan actividad física 2 veces por semana (50\%), dos que brindan 3 veces por semana (17\%) y otras dos que tienen una frecuencia de 5 veces por semana (17\%).

En ocho de doce instituciones (67\%) la actividad brindada tiene una duración de 60 minutos, en una institución la duración es de 90 minutos, en otra de 50 minutos y en las dos restantes es de 30 minutos.

La adherencia de los pacientes a la actividad física, teniendo en cuenta todas las instituciones (públicas y privadas), es media en un $67 \%$, alta en un $25 \%$ y baja en un $8 \%$. En las instituciones públicas la adherencia es $33,3 \%$ baja, $33,3 \%$ media y $33,3 \%$ alta. En las instituciones privadas la adherencia es $78 \%$ media y $22 \%$ alta.

Todos los participantes fueron consultados si conocían algún beneficio sobre la realización de actividad física en pacientes psiquiátricos. De diecisiete encuestados, quince respondieron que sí conocen algún beneficio, dentro estos, uno respondió que conoce beneficios pero agregó que no está informado de cuales son, más allá de mejorar el sedentarismo. De los dos restantes, uno respondió que no conoce ningún beneficio, mientras que el otro encuestado no respondió esa pregunta.

Diez (83\%) de los doce encuestados pertenecientes a instituciones que brindan actividad física respondieron que notan resultado terapéutico psiquiátrico en los pacientes que participan de la actividad, mientras que dos contestaron que no (17\%). La descripción de los resultados observados por los encuestados fueron mejorías anímicas y cognitivas, mejoría global, cohesión grupal, recreación, menor aumento de peso, mejorías volitivas, autovalidación, disminución de la ansiedad. De los dos encuestados que contestaron que no los observan, uno de ellos es el mismo encuestado que respondió que no conoce ningún beneficio de la realización de actividad física en pacientes psiquiátricos, mientas que el otro respondió que sí los conoce.

En el caso de responder que no se brinda actividad física a los pacientes internados en la institución a la que representa se les preguntó los motivos por los cuales consideran que no se hace. Entre las respuestas se encuentran la falta de recursos y el hecho de no considerarla una actividad de importancia en el tratamiento psiquiátrico.

Asimismo, uno de los encuestados respondió que en la institución que representa sí se brinda actividad física, pero en la sección de comentarios agregó que esta actividad era llevada a cabo por alguien que no percibía salario y dependía de la iniciativa de personal no especializado en actividad física. Agregó además que los motivos serían por falta de recursos y por no considerarla de importancia terapéutica. Dicho encuestado representaba a una institución pública.

Un representante de una institución pública en la que sí se brinda actividad física comentó que no todos los pacientes realizan actividad física ya que los diferentes servicios dentro de esa institución no estimulan la actividad física de la misma manera.

Otro representante de una institución pública en la que no se brinda actividad física agregó que el jefe de internación de dicha institución considera inconve- 
niente que los pacientes realicen actividad física, por motivos que el encuestado desconoce.

\section{Discusión}

Los resultados del presente trabajo exponen que en la mayoría (71\%) de las instituciones con internación psiquiátrica para adultos de la Ciudad Autónoma de Buenos Aires se brinda actividad física a los pacientes durante el período de internación. También expone que existe una importante diferencia en la implementación de la actividad física como recurso terapéutico ya sea que se trate de institución pública o privada.

Se observan diferencias en la participación de los pacientes en la actividad física dependiendo que se trate de una institución pública o privada, observándose mayores tasas de participación en las instituciones privadas, que a su vez son las que brindan actividad física en mayor proporción.

Las tasas de adherencia más bajas se observan en las instituciones públicas que son a la vez las que brindan actividad física en menor proporción. Estos resultados podrían sugerir que la adherencia de los pacientes a la realización de actividad física podría estar relacionada con la oferta de este recurso terapéutico a los pacientes por parte de las instituciones, lo que a su vez podría estaría relacionado con la importancia que le asignan las instituciones/profesionales a este recurso. Sería interesante profundizar en futuras investigaciones sobre los motivos de estas diferencias en la adherencia, para poder dilucidar si estaría relacionado con los conocimientos sobre este recurso que poseen los profesionales y las instituciones, con la manera en que se realiza la indicación a los pacientes o con qué otra razón.

Si bien la afectación de voluntad presente en los pacientes psiquiátricos puede ser una limitación para la adhesión de los pacientes a la actividad física, el rol de la institución y del profesional tratante a la hora de realizar la indicación y de ofrecerlo como herramienta terapéutica podría ser relevante.

La motivación de los pacientes para realizar actividad física puede verse favorecida con profesionales e instituciones que alienten su participación en dichas actividades, como así también mediante la selección de actividades que le sean placenteras al individuo en tratamiento.

Otras estrategias para aumentar la adhesión podrían ser variabilidad de oferta de las actividades y principalmente que exista personal capacitado en preparación física para llevar adelante este abordaje.

\section{Comentarios}

Durante el desarrollo del presente trabajo el Instituto de Neurociencias de Buenos Aires (INEBA) cerró el servicio de internación. Dado que la encuesta fue enviada con anterioridad al de cierre de la institución, los resultados fueron incluidos.

\section{Limitaciones}

Algunas instituciones poseen diferentes servicios con funcionamiento diverso entre ellos, la repuesta de un solo servicio puede no ser representativa. Se necesitaría un diseño que contemple los diferentes servicios y las diferencias entre ellos.

Con el fin de realizar una encuesta anónima no se conoce con exactitud qué instituciones respondieron la encuesta. Si bien se les pidió a los participantes que informaran una vez que la hubiesen completado, no quedó registro fehaciente de qué instituciones fueron representadas y cuáles no. En especial cuando un participante no informaba su participación y se buscaba otro representante para esa institución, sin poder conocer con exactitud si el primero no completó la encuesta o si la completó y no lo informó.

Los resultados podrían tener el sesgo de que los participantes fueron todos conocidos directa o indirectamente por la autora y no ser elegidos al azar.

A su vez cada representante brinda información sobre sus opiniones y conocimientos que pueden no corresponder con los de la institución a la que representa.

Conflictos de intereses: la autora declara no tener conflictos de intereses.

Agradecimientos: la autora agradece a todos y a cada uno de los participantes de la encuesta y a las personas que le brindaron su ayuda para contactarse con los participantes. Quiere agradecer al Dr. Juan Cristóbal Tenconi y especialmente a la Dra. Viviana Peskin cuya ayuda ha sido de vital importancia para la realización del presente trabajo.

\section{Referencias bibliográficas}

Adams, D. J., Remick, R. A., Davis, J. C., Vazirian, S., Khan, K. M. (2015). Exercise as medicine-the use of group medical visits to promote physical activity and treat chronic moderate depression: a preliminary 14-week pre-post study. BMJ Open Sport Exerc Med, 1(1):e000036. doi:10.1136/ bmjsem-2015-000036

Brand, S., Colledge, F., Beeler, N., et al. (2016). The current state of physical activity and exercise programs in German-speaking, Swiss psychiatric hospitals: results from a brief online survey [published correction appears in Neuropsychiatr Dis Treat. 2020 Jul 23;16:1765]. Neuropsychiatr Dis Treat, 12:1309-1317. doi:10.2147/NDT.S107313 
Ehrbar, J., Brand, S., Colledge, F., et al. (2018). Psychiatric In-Patients Are More Likely to Meet Recommended Levels of Health-Enhancing Physical Activity If They Engage in Exercise and Sport Therapy Programs. Front Psychiatry, 9:322. doi:10.3389/fpsyt.2018.00322

Firth, J., Carney, R., French, P., Elliott, R., Cotter, J., Yung, A. R. (2018). Long-term maintenance and effects of exercise in early psychosis. Early Interv Psychiatry, 12(4):578-585. doi:10.1111/eip.12365

Kandola, A., Hendrikse, J., Lucassen, P. J., Yücel, M. (2016). Aerobic Exercise as a Tool to Improve Hippocampal Plasticity and Function in Humans: Practical Implications for Mental Health Treatment. Front Hum Neurosci, 10:373. doi:10.3389/fnhum.2016.00373

Kandola, A., Vancampfort, D., Herring, M., et al. (2018). Moving to Beat Anxiety: Epidemiology and Therapeutic Issues with Physical Activity for Anxiety. Curr Psychiatry Rep, 20(8):63. doi:10.1007/s11920-018-0923-x

Kim, Y. S., Song, B. K., Oh, J. S., Woo, S. S. (2014). Aerobic exercise improves gastrointestinal motility in psychiatric inpatients. World J Gastroenterol, 20(30):10577-10584. doi:10.3748/wjg.v20.i30.10577

Kimhy, D., Vakhrusheva, J., Bartels, M. N., et al. (2015). The Impact of Aerobic Exercise on Brain-Derived Neurotrophic Factor and Neurocognition in Individuals With Schizophrenia: A Single-Blind, Randomized Clinical Trial. Schizophr Bull, 41(4):859-868. doi:10.1093/schbul/sbv022
Loh, S. Y., Abdullah, A., Abu Bakar, A. K., Thambu, M., Nik Jaafar, N. R. (2015). Structured Walking and Chronic Institutionalized Schizophrenia Inmates: A pilot RCT Study on Quality of Life. Glob J Health Sci, 8(1):238248. doi:10.5539/gjhs.v8n1p238

Morris, J. K., Vidoni, E. D., Johnson, D. K., et al. (2017). Aerobic exercise for Alzheimer's disease: A randomized controlled pilot trial. PLoS One, 12(2):e0170547. doi:10.1371/journal.pone.0170547

Netz, Y. (2017). Is the Comparison between Exercise and Pharmacologic Treatment of Depression in the Clinical Practice Guideline of the American College of Physicians Evidence-Based? Front Pharmacol, 8:257. doi:10.3389/fphar.2017.00257

Song, B. K., Kim, Y. S., Kim, H. S., Oh, J. W., Lee, O., Kim, J. S. (2018). Combined exercise improves gastrointestinal motility in psychiatric in patients. World J Clin Cases, 6(8):207-213. doi:10.12998/wjcc.v6.i8.207

Svatkova, A., Mandl, R. C., Scheewe, T. W., Cahn, W., Kahn, R. S., Hulshoff Pol, H. E. (2015). Physical Exercise Keeps the Brain Connected: Biking Increases White Matter Integrity in Patients With Schizophrenia and Healthy Controls. Schizophr Bull, 41(4):869-878. doi:10.1093/schbul/sbv033 